attend hospice multidisciplinary community team meetings. Patients with complex cancer pain syndromes are discussed and proactive joint reviews and procedures arranged where appropriate. Joint consultation with the chronic pain consultant and palliative care team is emphasised to ensure that all options are explored pre-procedure and follow-up is safe postprocedure. It is anticipated that case reviews presented back to the clinical teams will encourage referrals and contribute to ongoing education. In addition, over the first year of the service, the systems to support hospice-based spinal infusion services for intractable cancer pain will be established.

Evaluation A service evaluation will assess numbers of patients identified for discussion, assessment and intervention; clinical team confidence and knowledge; and case note review of perceived patient benefits.

\section{P-119 A RETROSPECTIVE STUDY EXPLORING SUBCUTANEOUS LEVETIRACETAM USE AND PRACTICE WITHIN THE WEST MIDLANDS}

Matthew Dore, Sharon Twigger, Kate Holyhead, Clare Marlow. NHS, Compton Hospice, WM CARES (West Midlands Collaborative Actioning Research in End-of-life and Supportive Care)

\subsection{6/bmispcare-2017-hospice. 144}

Background Levetiracetam is increasingly used subcutaneously to control seizure activity in selected palliative patients, whereby seizure control is paramount without the same perceived sedative effect of subcutaneous benzodiazepines. Despite this becoming a well recognised approach the literature and quality evidence to support this remains sparse.

Aim To perform a retrospective audit exploring subcutaneous Levetiracetam use and practice, over the past year across the West-Midlands, comparing it to guidance in PCF5.

Method An electronic survey sent to all palliative care units and hospital teams across the West Midlands generated information from 31 cases where subcutaneous Levetiracetam had been used. Information gathered included seizure aetiology and type, antiepileptic history, delivery of Levetiracetam, side effects and effectiveness.

Results Seizure aetiology was heterogeneous; 50\% caused by space-occupying lesions. $42 \%$ of patients had seizures in the week prior to commencement and $58 \%$ of seizures were described as tonic-clonic. Nearly all (93\%) had anti-epileptics prescribed prior to commencement. The majority of Levetiracetam was delivered via a continuous subcutaneous infusion (92\%), the remaining given by bolus subcutaneous regimen. The mean dose on commencement was $1268 \mathrm{mg}$ (range $250 \mathrm{mg}-3000 \mathrm{mg}$ ) and $12 \%$ of infusions were titrated over time due to seizure activity. Levetiracetam was successfully mixed with morphine, midazolam, metoclopramide and dexamethasone with no issues reported. In 69\% cases concurrent midazolam administration was used, although the rationale varied and was not solely seizure related. $81 \%$ reported no side effects attributable to Levetiracetam, 16\% reported a local site skin reaction. No further seizures were documented in $70 \%$ whilst on subcutaneous Levetiracetam and $62 \%$ of subcutaneous Levetiracetam regimens continued till death.

Conclusion This study outlines current varied practice in the West-Midlands demonstrating the targeted patient group and practical issues in using subcutaneous Levetiracetam. Collating this information adds to the evidence base and will allow for composition of informed local guidelines.

\section{P-120 DESCRIBING ANTICIPATORY MEDICINE USE IN THE COMMUNITY AT THE END OF LIFE}

${ }^{1,2,3}$ Edward Rainbow, ${ }^{2}$ Christina Faull. ' Countesthorpe Health Centre, Leicester, UK; ${ }^{2}$ LOROS Hospice, Leicester, UK; ${ }^{3}$ Health Education East Midlands (HEEM), Leicester, UK

\subsection{6/bmispcare-2017-hospice.145}

Background Anticipatory Medicines (AM) are injectable medicines prescribed and administered to patients to alleviate symptoms at the End of Life (EOL). Whilst prescribing of these medicines is encouraged by the healthcare system in Leicester, Leicestershire and Rutland, the actual patterns of prescribing and administration of AM have not been documented locally.

Aim To describe the prescribing and usage of AM in the community, matching this to EOL symptoms experienced by patients.

Method A retrospective audit of 150 sets of patient notes was conducted at the start of 2017, comprising 50 deaths from one General Practice list, 50 deaths from Community Palliative Care Nursing teams, and 50 hospice discharges. Data included the main diagnosis at death; and details of prescription and usage per ampule of each AM.

Results General Practice data analysed thus far indicates:

- Death by primary diagnosis: Cancer 16/50 (32\%), Chronic Disease 5/50 (10\%), Frailty/Dementia 22/50 (44\%), Sudden Death 5/50 (10\%)

- AM issued by diagnosis: Cancer 10/16 (62\%), Chronic Disease $0 / 5$ (0\%), Frailty/Dementia 11/22 (50\%), Sudden Death $1 / 5(20 \%)$

- Median number of days AM issued and started [X] before death: Cancer 14[4], Chronic Disease (N/A), Frailty/Dementia 6[4], Sudden Death 11[6].

- Number of patients documented having EOL symptoms (13): Cancer 4, Chronic Disease (N/A), Frailty/Dementia 8, Sudden Death 1

- EOL symptoms experienced: Pain: 12 (Cancer 3, Frailty/ Dementia 8, Sudden Death 1), Breathlessness: 3 (Frailty/ Dementia 3), Agitation 2 (Cancer 1, Frailty/Dementia 1), Nausea 1 (Cancer 1), Vomiting 2 (Frailty/Dementia 2).

- Syringe Driver use: Cancer (2), Frailty/Dementia (5), Sudden Death(1)

- Total AM ampule usage [Number patients]: 0 [10 Cancer (3), Frailty/Dementia(7)], 0-9 [8, Cancer (2), Frailty/ Dementia(6)], 10-19 [2, Cancer (1), Sudden Death (1)], 20-49, [2, Frailty/Dementia (2)], $\geq 50$ [1, Cancer (1)]

Conclusions Patients with frailty/dementia have EOL symptoms similar to cancer, but with greater numbers in the community. AM prescription occurs a few days before their use. The data regarding Chronic Disease deaths in the community is limited in this audit. Further work is needed to analyse all datasets.

\section{P-121 A THEMATIC ANALYSIS OF ANTICIPATORY MEDICINE USE IN THE COMMUNITY AT END OF LIFE}

1,2,3 Edward Rainbow. ' ${ }^{1}$ Countesthorpe Health Centre, Leicester, UK; ${ }^{2}$ LOROS Hospice,
Leicester, UK; ${ }^{3}$ Health Education East Midlands (HEEM), Leicester, UK 10.1136/bmjspcare-2017-hospice. 146

Background Anticipatory Medicines (AM) are injectable medicines prescribed and administered for patients to alleviate symptoms at the End of Life (EOL). Whilst prescribing of these medicines is encouraged by the healthcare system in 